\title{
Chromato-Polarographic Studies. XVII. The Analysis of Mixture of 2,4-Dinitrophenylhydrazones of Aliphatic Aldehydes and Ketones
}

\author{
By \\ Wiktor Kemula, Karol Butkiewicz and Danuta Sybilska \\ Department of Inorganic Chemistry, University, Warszawa \\ Department of Physicochemical Analytical Methods, Institute of Physical Chemistry, \\ Polish Academy of Science, Warszawa
}

(Received Nov. 5, 1962)

An increasing interest for natural and synthetic aldehydes and ketones has caused that a significant number of analytical papers on this subject was recently published.

Until now some specific reactions of individual compounds were used in the classical organic analysis ${ }^{12}$. Aldehydes were also determined using some instrumental methods, $e$. g. polarography ${ }^{2}$. All these methods are non-selective and cannot be used in investigation of mixtures.

Recently adsorption chromatography, partition chromatography on carrier and paper, ion-exchange chromatography and gas-liquid chromatography were used for separation of aldehydes and ketones ${ }^{3)}$. Attempts were made to separate these compounds as their derivatives, viz. semicarbazones ${ }^{4}$, hydroxamic acid derivatives ${ }^{5}$, but highly promising results were obtained using 2,4-dinitrophenylhydrazones. This procedure has been described by many authors, who applied, among others, also reverse phase partition chromatography ${ }^{6-10}$. In this paper the chromato-polarographic method was used for the analysis of mixture of aldehydes and ketones.

The chromato-polarographic method ${ }^{15}$ may be used for the study of species reducible at the mercury dropping electrode over the potential range, which is not too negative. Otherwise, either some indirect meth $\lrcorner$ ds should be used ${ }^{11,12)}$, or, with usually better result ; the polarographically inactive compounds should be transformed into reducible ones ${ }^{13}$.

Quantitative formation of stronly coloured, insoluble precipitates of 2,4-dinitrophenylhydrazones of carbonyl compounds ${ }^{14)}$ enables application of colorimetric or polarographic methods to the determination of aldehydes and ketones. Nevertheless both mentioned methods are poor in selectivity and cannot be used for the analysis of mixtures of mentioned compounds.

\section{Experimental}

Reagents, Equipment.-In the mixtures studied the following compounds were present: saturated aliphatic aldehydes $\mathrm{C}_{3}-\mathrm{C}_{7}$ and saturated aliphatic ketones $\mathrm{C}_{1}-\mathrm{C}_{4}$. The physical constants of all preparations have been determined, and they were in reasonable agreement with published values. All other reagents and solvents were of analytical or chemical purity.

The previously reported ${ }^{15}$ chromato-polarographic equipment was used. The diffusion current was recorded by polarographic or visual instruments. 
All chromatographic experiments were performed with solutions containing dissolved air at the temperature $20 \pm 1^{\circ} \mathrm{C}$.

Precipitation of 2,4-dinitrophenylhydrazones.- Precipitation of derivatives of carbonyl compounds was carried out according to Iddless and coworkers ${ }^{14}$. To the saturated solution of 2,4-dinitrophenylhydrazine in $2 \mathrm{~N}$ hydrochloric acid in $50 \%$ excess, the solution of ketones or aldehydes in $95 \%$ ethanol was dropwise added with vigorous stirring. The carbonyl compounds solution contained $0,01 \mathrm{~g}$ per $1 \mathrm{ml}$ of ethanol. To the solution with precipitate $50 \mathrm{ml}$ of $2 N$ hydrochloric acid was added and after 24 hours filtred through a sintered glass plate. The solution was washed with $100 \mathrm{ml}$ of $2 \mathrm{~N}$ hydrochloric acid and with water until a negative reaction for $\mathrm{Cl}$-ions was found in the filtrate. The precipitate was dried to the constant weight at $100-110^{\circ} \mathrm{C}$. The precipitated 2,4-dinitrophenylhydrazones were then dissolved in a given volume of the mobile phase solution and $0,5 \mathrm{ml}$ of this solution, containing approximately $2 \mathrm{mg}$ of hydrazones, was placed on the top of the column.

Preliminary investigation of the separation system.- In the preliminary experiments some attempts were made to separate 2,4-dinitrohydrazones on ion-exchange columns using the salting out technique. Such columns are persistent and enable to change the mobile phase composition. Ion-exchange resins Dowex 50 and Amberlite IRG 200 and as a mobile phase aqueous buffered solutions of dimethylformamide with the pH from 5.57 to 4.05 have been used.

These experiments were unsuccessful. The studied compounds were bound on the ion exchanger column in spite of changes in $\mathrm{pH}$ and dimethylformamide concentration. No difference in chromatographic partition coefficients was observed. All components appeared simultaneously in the eluate.

In further research the reversed phase partition chromatography was used. Considering the low solubility of 2,4-dinitrophenylhydrazones in water it was necessary to use solutions containing a high percentage of organic solvent. The carrier rubber, prepared after Kemula and Butkiewicz, ${ }^{16)}$ has been used. Systems containing cyclohexane, heptane and isooctane as a stationary phase, and aqueous solutions of methanol, ethanol and isopropanol at various concentrations as a mobile phase, have been studied. In all cases serious disturbances in polarographic recording due to high content of organic solvents in the mobile phase have been observed. Finally success was attained when aqueous solutions of dimethylformamide as a mobile phase even at $90 \%$ concentration was used.

\section{Separation of 2,4-dinitrophenylhydrazones of saturated aliphatic ketones.}

Acetone, methyl ethyl ketone, methyl propyl ketone and methyl butyl ketone have been used. The stationary phase consisted of $2,5 \mathrm{~g}$ of rubber swollen with $2,5 \mathrm{ml}$ of cyclohexane, and the mobile phase of $3 \mathrm{M}$ KSCN in $80 \%$ aqueous dimethylformamide. Both phase were mutually saturated. The column height was $37,0 \mathrm{~cm}$, and its internal diameter $0,6 \mathrm{~cm}$. The diffusion current intensity was measured at the constant potential $-1,3 \mathrm{~V}$ $v s$. pool. The rate of elution was $7 \mathrm{ml} /$ hour. The samples used had the following composition : $1,0 \mathrm{ml}$ containing an aldehyde mixture at the concentration about $10^{-3} \mathrm{M}$. A complete separation of 2,4-dinitrophenylhydrazones of four investigated ketones has been obtained. Besides, 2,4-dinitrophenylhydrazine appears in a separate peak. This is of primary importance, because there is no need of very careful separation of the excess of 
the reagent used in the course of precipitation. The precipitated hydrazones may be immediately filtered, dried and dissolved in the mobile phase solution. Therefore, the time of an experiment did not exceed 6 hours. The zero current fluctuations in the studied system were insignificant and had no influence on the results.

Separation of 2,4-dinitrophenylhydrazones of saturated aliphatic aldehydes.

As an example we have separated propionaldehyde, $n$-butylaldehyde, $n$-valeraldehyde and $n$-heptaldehyde. As the stationary phase $1,8 \mathrm{~g}$ of rubber swollen with $1,8 \mathrm{ml}$ of isooctanol, as a mobile phase 0,1 $M \mathrm{KCl}$ in $70 \%$ aqueous dimethylformamide were used. The column height was $20,5 \mathrm{~cm}$, and its diameter $0,6 \mathrm{~cm}$. The diffusion current intensity was, as previously, recorded at $-1,3 \mathrm{~V} v s$. pool. The rate of elution was $6 \mathrm{ml}$ per hour. The samples had the following composition: an aldehyde mixture at the concentration about $10^{-3} \mathrm{M}$. The sample volume was $1,0 \mathrm{ml}$.



Fig. 1. Separation of 2,4-dinitrophenylhydrazones of aliphatic ketones by reversed phase partition chromatography.

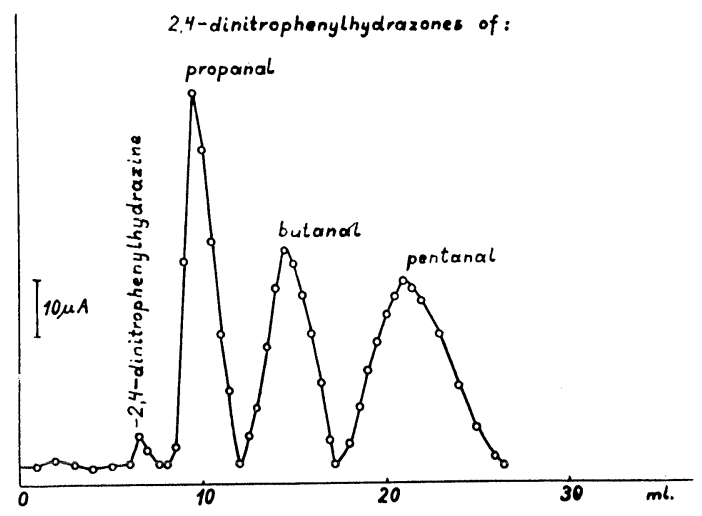

Fig. 2. Separation of [2,4-dinitrophenylhydrazones of aliphatic aldehydes by reversed phase partition chromatography.

In this case a complete separation of 2,4-dinitrophenylhydrazones of the above mentioned aldehydes was obtained, and, as previously, 2,4-dinitrophenylhydrazine came out in the eluate at the very beginning. The total time of analysis was in this case 3 hours.

The elution curves, shown in Fig. 2 are relatively sharp and symmetrical. Under these conditions the elution from the column is quantitative. The obtained accuracy is about $\pm 5 \%$.

\section{Separation of free aldehydes.}

The fact that free saturated aliphatic aldehydes are polarographically reducible indicates that separation of these compounds can be performed directly without "polarographic activation" through the reaction with 2,4-dinitrophenylhydrazine.

No success was obtained by means of partition chromatography. Owing to the extremely negative reduction potential $(-2,0 \mathrm{~V})$ of aldehydes in the systems studied, no usual polarographic supporting electrolyte could be used. The zero drift were rather large. No convenient solvent system could be found.

In such a situation reasonably good results should be expected by means of adsorption chromatography. Among the various adsorbents investigated only cellulose acetate was 
found to be suitable for aldehyde separation under chromatographic conditions. As a mobile phase aqueous and alcoholic solutions of tetraethylammonium iodide were used. In Fig. 3 is shown an elution analysis of four aldehydes.

The separation was carried out in a system, the stationary phase of which was prepared of $1,0 \mathrm{~g}$ of cellulose acetate, with the $0,075 \mathrm{~mm}$ diameter of individual particle, and the mobile phase of $0,1 \mathrm{M}$ tetraethylammonium iodide in $30 \%$ aqueous methanol. The column height was $7,5 \mathrm{~cm}$, its internal diameter $0,6 \mathrm{~cm}$.

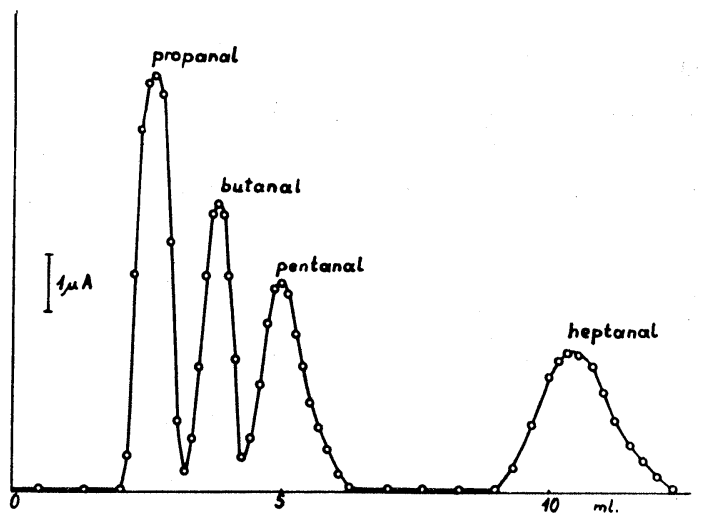

Fig. 3. Separation of aliphatic aldehydes by adsorption chromatography.

The sample volume of about $0,5 \mathrm{ml}$, containing an aldehyde mixture at the concentration about $10^{-3} M$, was placed on the top of the column and washed with the above described solution. The diffusion current was measured at $-2,0 \mathrm{~V} v s$. pool. The rate of elution was $7 \mathrm{ml}$ per hour.

As shown by Fig. 3, the separation is satisfactory for propionaldehyde, $n$-butylaldehyde, $n$-valeraldehyde and heptaldehyde. It should be supposed that hexylaldehyde will appear in the eluate between valeraldehyde and heptaldehyde without influence on the result of determination of the other aldehydes. The duration of an analysis requires about 2 hours.

\section{Summary}

The chromato-polarographic method have been used for the analysis of aldehydes and ketones after their quantitative reaction with 2,4-dinitrophenylhydrazine. Considering the low solubility of 2,4-dinitrophenylhydrazones in water, it is necessary to use aqueous solutions containing high percentage of dimethylformamide for the partition chromatography on rubber columns. Even in $80 \%$ concentration dimethylformamide does not disturb the polarographic measurements. The elution curves are symmetrical, the substances are eluted quantitatively seaprated and the accuracy of determation is equal $\pm 5 \%$. Due to chromatographic separation of the excess of 2,4-dinitrohydrazine there is no need for its complete separation before running the chromatopolarogram. It was found that the concentration of dimethylformamide higher than $80 \%$ interferes seriously at the polarographic measurements and decreases the durability of the column prepared from the rubber carrier.

Furthermore, it is found that the first members of the homologous series of saturated aliphatic aldehydes can also be separated by means of an adsorption column prepared of cellulose acetate, without transformation into 2,4-dinitrophenylhydrazones.

\section{References}

1) Mitschell, J.Jr., I.M. Kolthoff, E.S. Proskauer, A. Weissberger, Organic Analysis, Vol. 1, Interscience, New York, 1953, p. 243.

2) Petrowa, L.N., Novikowa, E.N., Zhur. Priklad. Khim., 28, 219 (1955). 
3) Lederer E., Lederer M., Chromatography, Elsevier, London, 1957, pp. 167-169.

4) Di Modica, G., Spriano, C., Ann. Chim. (Rome), 41, 64 (1951).

5) Uno, H., Koyama, A.,J. Ferment. Technol. (Japan), 29, 219 (1951).

6) Kostir, J.V., Slavik, K., Collection Czechoslov. Chem. Communs., 15, 17 (1950).

7) Rice, R.G., Keller, G.J., Kirchner, J.G., Anal. Chem., 23, 194 (1951).

8) Elis, Rex, Gaddis, A.M., Currie, G.T., Anal. Chem., 30, 475 (1958).

9) Kramer, P.J.G., Van Duin, H., Rec. trav. chim., 73, 63 (1954).

10) Howard, G.A., Tatschell, A.R., Chem. and Ind., 219 (1954).

11) Kemula, W., Roczniki Chem., 29, 653 (1955).

12) Kemula, W., Stachurski, Z., unpublished results.

13) Kemula, W., Witwicki, J., Roczniki Chem., 29, 1153 (1955).

14) Iddles, H.A., Low, A.W., and others., Ind. Eng. Chem. Anal. Ed., 11, 102 (1939).

15) Kemula, W., Roczniki Chem. 26, 261 (1952) ; Przem. Chem. 33, 453 (1954); Proceedings of the International Symposium on Microchemistry, Birmingham, 1958, p. 258, Pergamon ; Progress in Polarography, p. 397, Interscience, 1962, New York.

16) Kemula, W., Butkiewicz, K., Chemia Anal., 1, 56 (1956).

17) Kemula, W., Sybilska, D., Geisler, J., Chemia Anal. 1, 36 (1956). 